\title{
LAS LEYES DE LA GUERRA (EXISTENCIA Y APLICABILIDAD)
}

TERESA SANTIAGO

\author{
DEPaRTamento de Filosofía de la División de Ciencias Sociales y Humanidades
}

UNIVERSIDAD AUTÓNOMA METROPOLITANA-IZTAPALAPA

Inter arma silent leges:

"En tiempos de guerra la ley guarda silencio"

Si bien es cierto que la preocupación en torno a la posible regulación de los conflictos armados ha sido una constante a lo largo de la reflexión acerca de la naturaleza de la guerra y su posible justificación, es a partir de las dos guerras mundiales ocurridas en el presente siglo cuando surgen en el panorama jurídico internacional conceptos tales como crímenes de guerra, crímenes contra la paz y contra la humanidad. El hecho de que estos conceptos hayan aparecido en la jerga corriente cuando se enjuician y valoran los conflictos armados indica que se admite la existencia de lo que podríamos llamar un conjunto de normas o leyes de guerra. Empero, cuando intentamos definir el concepto de "leyes", así como señalar cuáles serían los contenidos de éstas, nos encontramos con dificultades que rebasan el mero campo de la legislación o de los códigos particulares, así se hace evidente que nos enfrentamos a un problema que compete en gran medida a la reflexión filosófica. El ensayo que se presenta a continuación tiene la finalidad de aclarar algunas de las dificultades que se vinculan con el problema de la existencia y la posible aplicación de esas leyes, justamente, desde la perspectiva de un análisis filosófico. Con lo cual estamos descartando una aproximación jurídica o histórica, aunque, obviamente, alguna alusión tendrá que hacerse a este tipo de factores. El orden en que se presentan los distintos temas y argumentos necesarios para dar inteligibilidad a la exposición es el siguiente: en primer lugar habrá que decidir si la guerra es un asunto pertinente para la valoración moral; en segundo lugar se abordará el problema de cómo las leyes de la guerra han tenido que enfrentarse y, en ocasiones, ajustarse a los requerimientos de la llamada "doctrina militarista"; por último, se discutirán algunas cuestiones centrales en torno a los problemas que implica la aplicación de las leyes de guerra y se ofrecerán algunas conclusiones al respecto. 


\section{La guerra como ámbito de la moralidad}

A lo largo de la historia de la humanidad la guerra ha sido objeto de múltiples y muy variadas consideraciones. Desde las creencias del hombre común que se considera absolutamente autorizado a opinar y juzgar sobre esta actividad omnipresente en el terreno de lo humano, hasta las elaboradas reflexiones filosóficas, pasando por la literatura, la pintura y demás manifestaciones artísticas, la guerra es un asunto frente al cual difícilmente podemos mantenernos indiferentes. Son, pues, muchas las maneras de aproximarnos a la guerra y de interpretarla. Pero ninguna tarea ha sido tan compleja como decidir si la guerra compete genuinamente al área de la moralidad y en qué terminos. Hay quienes han considerado que ésta nunca puede justificarse moralmente; baste recordar a Tolstoi y a Gandhi, quienes enarbolaron la idea de un pacifismo radical en virtud del cual siempre es preferible sufrir la violencia a ejercerla. En contraste, también se da el caso de autores a favor de la idea de que la guerra, aun siendo un mal, es inevitable porque es uno de los mecanismos que hacen posible el desarrollo de las sociedades. Ligada a esta idea subyace la convicción de que los logros económicos y políticos que se obtienen con la guerra no se pueden alcanzar por otros medios. Apoyados en razones de este tipo y en otras similares, muchos autores han pugnado por dirigir las discusiones hacia la posible regulación de la guerra, i.e., hacia la probabilidad de encontrar mecanismos que garanticen el recurso legítimo a la guerra así como una conducta apegada a los principios morales y humanitarios.

Aunque no todos los filósofos comprometidos con semejante tarea pueden ser integrados a la llamada "doctrina de la guerra justa", cuya paternidad intelectual casi siempre se atribuye a San Agustín, ${ }^{1}$ es indudable que dicha tradición marcó de una vez y para siempre los dos ámbitos de competencia en los que se han desarrollado los distintos argumentos: la justificación de la guerra como recurso o jus ad bellum y la cuestión de la justicia en la guerra, i.e., la conducta de guerra o jus in bello. Siendo cuestiones lógicamente independientes, de cualquier manera mantienen vínculos que distan mucho de ser aproblemáticos. Por ejemplo, en muchas ocasiones se ha defendido la idea de que una guerra se justifica cuando se lleva a cabo con medios justos a pesar de que no pueda justificarse, en principio, la causa por la que fue emprendida. También es necesario apuntar que el desarrollo de ambas líneas o tradiciones no ha sido homogéneo. La parte de la doctrina conocida como jus ad bellum se desarrolló más en un principio (siglo xrv) que la del jus in bello, la que desde los siglos xvI y

1 Aunque fue Aristóteles, según observa Michael Walzer en su obra Just and Unjust Wars, quien acuñó la expresión "guerra justa" para referirse a las guerras emprendidas por los griegos con la finalidad de afianzar su poderío político y proveerse de esclavos. 
xVII ha gozado de un desarrollo importante al margen de la primera doctrina. Ciertamente esto no es casual. Derivada del pensamiento cristiano de los primeros siglos, la guerra fue vista por San Agustín y más tarde por los filósofos escolásticos, como el medio por el cual podían cumplirse los designios de Dios, en contra de una sociedad pagana en la que imperaba un orden injusto: "Quien desee comprender la teoría de la justum bellum, debe comprender en los escritos de San Agustín, la similitud entre su crítica a las virtudes personales de los paganos y su crítica a la justicia social en cualquiera de las naciones e imperios de su tiempo. .. "2 De esta manera, la causa por la cual se emprende una guerra tiene una importancia central: una guerra justa es una guerra en donde principios fundamentales de la moral cristiana como "no matarás" pueden ser quebrantados si con ello se acerca el hombre al ideal divino, a la Ciudad de Dios. Es así como la doctrina del jus ad bellum va ligada a cierta concepción del orden de las cosas, un orden divino, vigente hasta el siglo $\mathrm{xv}$, aproximadamente. $Y$ aunque esta misma doctrina ha conocido también versiones secularizadas que la han mantenido presente en las discusiones, es cierto que no ha vuelto a tener el vigor de sus primeros tiempos. Por su parte, la doctrina del jus in bello adquirió importancia y autonomía con el surgimiento del "espíritu nacional" y de los ejércitos regulares. Como veremos más adelante, con el nacimiento de las naciones modernas se neutralizó la preocupación por la causa justa de guerra a favor de una doctrina de la proporción entre los objetivos de la guerra y los medios para conseguirlos, misma que se ajustó a la lógica del jus in bello. Muchas de las discusiones más intensas de las últimas décadas sobre el tema de armamentos nucleares, guerras totales y limitadas, etc., atañen, justamente, a esta parte de la doctrina.

Más allá de los complejos problemas que se han suscitado en cualquiera de las dos líneas de discusión mencionadas anteriormente, es claro que esta larga tradición filosófica ha considerado que la guerra sí es un tema de competencia moral, esto es, que no puede soslayarse el problema de la normatividad de la guerra dada su presencia innegable y, hasta ahora, inevitable en la vida de los hombres. Además de esta razón, pueden traerse a colación otras tanto o más convincentes. Sin embargo, un argumento importante a favor de que la guerra sí es un asunto de la moralidad que no siempre se ha hecho explícito es el siguiente: aunque en otras épocas la guerra no fue vista como un mal, hace tiempo (por lo menos durante el siglo por concluir) que esta visión ha cambiado. En cierto sentido todos estaríamos de acuerdo en que la guerra es un mal. En todo caso, lo importante es ir más allá de esta constatación y percatarnos de que tal afirmación lo que puede estar

2 Paul Ramsey, "The Just War According to St. Augustine", en J.B. Elshtain (ed.), Just War Theory, p. 8. 
indicando es, justamente, la necesidad de ubicar a la guerra en el terreno de la normatividad: si aceptamos que la guerra es un mal, tendremos que aceptar como una tarea urgente restringirla lo más posible, en tanto recurso, y vigilar que se lleve a cabo dentro de límites razonables de violencia. En otras palabras, aceptar que la guerra es un mal implica hacernos conscientes de que es necesario poner límites a algo que es inevitablemente nocivo. Es cierto que hay otra conclusión que podría desprenderse de la misma consideración original: si la guerra es un mal, lo moralmente correcto es luchar por evitarla en lugar de reglamentarla. Hay autores que incluso han pensado que es inmoral hablar de normas y leyes de guerra. Pero si uno no quiere comprometerse con una postura radical como la del pacifista, por considerarla cercana a una forma de utopismo, una postura más moderada podría encontrar apoyo en el argumento que he señalado en primera instancia, ya que al mismo tiempo que permite mantenerse dentro de una concepción realista acerca de la guerra, no renuncia a su posible legislación.

Podemos señalar los puntos que sintetizan el contenido de la doctrina de la guerra justa tal como podría ser enunciada en la actualidad, con el fin de tener más claro el contenido de la misma.

1. Dado que la violencia es indeseable, una guerra justa tendrá que ser aquella que se dé en casos excepcionales, esto es, sólo cuando se trate de una guerra defensiva o bien cuando se intente resarcir de algún mal mayor, o injusticia, a pueblos y grupos humanos que no pueden hacerlo por sí mismos.

2. Una guerra es una acción que sólo puede decidir llevar a cabo el Jefe de estado o la autoridad competente.

3. Una guerra es justa sólo si las metas que se pretenden alcanzar con ella son legítimas, esto es, si se declara con base en una intención correcta.

4. Una guerra es justa si los fines que se pretenden alcanzar no pueden ser logrados por otros medios.

5. Una guerra es conducida justamente si además de ser el único medio para lograr ciertas metas legítimas, los medios con los que se lleva a cabo guardan proporción con los fines que se quieren alcanzar y se hace una discriminación sobre los objetivos y personas afectadas.

6. Los ciudadanos que deben lealtad a su país tienen el compromiso moral de participar en las guerras que los soberanos o el estado emprende si y sólo si ésta es conducida justamente.

7. Si las autoridades de un estado soberano emprenden guerras injustas de manera sistemática, los compromisos morales de lealtad de los 
ciudadanos hacia ese estado se rompen y pueden ser reemplazados por otro tipo de lealtades.

8. En casos extremos, los ciudadanos pueden tener el derecho moral de ir en contra de las autoridades de su propio estado como una manera de impedir que se persista en una guerra injusta.

Como ya se había indicado, los principios de la doctrina pertenecen a dos grupos distintos: los puntos 1 al 4 corresponden al jus ad bellum o causa justa de guerra y del 5 al 8 se refieren a la conducta y medios de guerrra o jus in bello. Unos y otros se alcanzan a reconocer en los códigos y manuales de guerra existentes desde épocas muy antiguas y también han nutrido los contenidos de los acuerdos del Derecho Internacional que ha intentado normar y regular las relaciones entre las naciones. No es nuestra intención llevar a cabo una revisión exhaustiva de dichos códigos y manuales particulares. Sin embargo, sí podemos hacer referencia a algunos de ellos con el propósito de constatar hasta qué punto reflejan las inquietudes del teórico o filósofo de la guerra y cómo éstas han tenido que sobrevivir a contrapelo de lo que se puede llamar la "doctrina militarista".

\section{Las leyes de guerra y la doctrina militarista}

Cuando en 1945 los Aliados se reunieron para formular las bases de lo que sería el juicio de Nuremberg en el que se juzgaría a los principales dirigentes de las naciones del Eje, los encargados de llevar a cabo las sesiones de aquel tribunal dieron por sentado que había antecedentes históricos suficientes para poder hablar de un conjunto de reglas o "leyes de guerra", mismas que habían sido violadas por Alemania y Japón. Dichos antecedentes podían encontrarse en las resoluciones de los Congresos de la Haya, los cuales permitían hablar de "crímenes de guerra", en el Congreso internacional de la Cruz Roja realizado en Génova que permitía establecer "crímenes contra la humanidad" y, finalmente, los llamados "crímenes contra la paz" por el pacto de Paz de París del año 1928. Se consideran "crímenes de guerra" toda violación a las leyes de la guerra a las que se refiere la parte de la doctrina de la guerra justa sobre medios y conducta de guerra o jus in bello, v.g., uso de armas prohibidas, maltrato a prisioneros y a civiles en países ocupados y devastación no justificada por necesidades militares. "Crímenes contra la humanidad" son aquellos actos dirigidos a diezmar o exterminar a algún grupo en particular por razones políticas, étnicas o religiosas, crímenes que quedan recogidos en la palabra "genocidio" también considerada por el jus in bello. Por último se consideran "crímenes contra la paz" los que comete una nación para iniciar una guerra agresiva y que, por tanto, quedarían considerados en la parte de la doctrina de la causa justa o jus ad bellum. Si 
dicha clasificación resulta satisfactoria es algo sobre lo que tendremos que opinar más adelante. Primero habría que considerar parte de la trayectoria histórica de las así llamadas "leyes de guerra".

Un dato del cual podemos partir sin necesidad de mayores justificaciones es el siguiente: la guerra es una actividad regulada, esto la distingue de la violencia caótica que ocasionalmente surge entre los grupos humanos para vengarse o resarcirse de alguna ofensa recibida: los varones del pueblo $X$ que armados de palos y piedras irrumpen en el pueblo $Y$ para tomar venganza porque alguna de sus mujeres ha sido violada o porque han robado su ganado; las acciones de un pueblo para hacerse justicia "por propia mano" en contra de alguna autoridad que ha actuado abusivamente, etc., no pueden considerarse propiamente hablando "guerras". En efecto, la guerra, como la cacería, o el deporte en general, es una forma de actividad humana en la que participan un buen número de personas y que funciona de manera más o menos organizada de acuerdo con normas que establecen ciertos límites. Ahora bien, no todas las reglas relacionadas con la guerra tienen un carácter moral. Buena parte de los códigos y manuales militares tienen como objetivo fijar los niveles de rango y jerarquía a través de reglas disciplinarias cuya finalidad es la de convertir al soldado en un dócil pero firme ejecutante de las órdenes que vienen de sus superiores. Y aunque, como se verá más adelante, esto es relevante desde el punto de vista de la moral, sólo nos referiremos a aquellas que caen en la consideración de la doctrina de la guerra justa, misma que hemos tomado como marco teórico para nuestra discusión.

Algunas de las primeras reglas que se establecieron en relación con la guerra datan de la Edad Media y se refieren a la declaración formal de las hostilidades así como al trato cortés hacia el enemigo. Al respecto, no hay que olvidar que en esa época, la guerra era una actividad propia de la nobleza. "Cuanto más refinada y aristocrática es la cultura, más legalizado está el combate."3 Por ello no es casual que entre las culturas antiguas destaque China como la que con mayor denuedo se ocupó de establecer reglas de honor y humanidad para los combates. También en Occidente, desde la Edad Media hasta el siglo xviI, la guerra era concebida como una actividad propia de caballeros, esto es, regulada por normas estrictas relacionadas con el honor y el prestigio. Es mucho más fácil diezmar al enemigo que derrotarlo en una lucha justa y reglamentada, como el propio Gallois afirma: "el respeto a los convencionalismos fundamenta el prestigio que se atribuye a la proeza. Una proeza es una acción valiosa y difícil que se cumple de acuerdo con un código, abiertamente fijado, que reduce considerablemente la libre iniciativa del héroe". ${ }^{4}$ Sin duda las guerras medievales tuvieron

3 Roger Gallois, La cuesta de la guerra, p. 32.

4 Ibid., p. 35. 
casos de crueldad y excesos, pero considerada de forma general, la guerra era una actividad que se concebía dentro de normas y reglas que intentaban fijar límites a las acciones violentas. Son muchas las referencias que pueden encontrarse en los códigos que regulaban las guerras caballerescas acerca del trato a prisioneros y a personas hors de combat. Éstas estaban dirigidas a señalar la deshonra que representaba, por ejemplo, ocupar iglesias, matar sacerdotes, niños, ancianos y mujeres, así como el empleo de ciertas armas como la ballesta. El principio de honorabilidad del guerrero era un valor sobreentendido más allá de las consideraciones militaristas.

Resulta interesante el hecho de que entre las guerras de los primeros siglos de nuestra era, fueran las guerras "santas", esto es, aquellas libradas en nombre de alguna fe religiosa, las que vieron obstáculos para lograr la victoria sobre el enemigo en estos intentos por regular y limitar las acciones bélicas. La misma doctrina de la guerra justa formulada por San Agustín es ambigua en este punto. Por un lado constituye el primer y más importante intento por justificar la guerra, es decir, por establecer límites morales a las acciones y, por el otro, establece que el soldado cristiano tiene el deber, como ciudadano y hombre de fe, de hacer todo lo posible por derrotar al enemigo, con mayor razón si se trata de un infiel: en el nombre de Dios podían cometerse toda serie de excesos y barbaridades. Esto pone de manifiesto que la guerra siempre existió ligada a la necesidad de su regulación en tanto recurso que puede emplearse, como también en cuanto a las acciones realizadas en ella. Pero al mismo tiempo, y esto es muy importante, la guerra siempre tuvo objetivos que cumplir, metas que muchas veces tuvieron que ser justificadas en nombre de una fe, un dios, una nación o un credo político.

Cómo fue transformándose la idea caballeresca de la guerra hacia una concepción netamente "militarista" de la misma, es un proceso sumamente complejo, pero el factor de cambio más evidente se encuentra quizás en el hecho de que la guerra se convirtió en un asunto en el que participaban ya no sólo algunos estratos sociales sino el pueblo en general. Con el surgimiento de los estados nacionales, nace el ciudadano y nace también el ejército regular, conformado por individuos sin importar la clase o la condición económica. Paradójicamente, el servicio de las armas se convierte en un instrumento de democratización de la sociedad, el individuo tiene ahora derechos y también deberes para con la nación a la que pertenece. La defensa de la nación no es exclusiva de unos cuantos sino que es deber de todo ciudadano porque lo que se pone en riesgo es su propia condición de hombre libre. "La naturaleza de la guerra pronto cambia: las luchas que enfrentan reinos combatientes son violentas y sangrientas. No son simples rivalidades de prestigio. Es el choque de naciones enemigas [...] se desarrolla una moral de potencia que 
acompaña o reemplaza las costumbres caballerescas de antaño en vigor." ${ }^{5}$ Es así como con el nacimiento de las naciones modernas la doctrina militarista inicia su proceso de consolidación. El surgimiento de un "espíritu nacional" llevó a un abandono gradual de la preocupación acerca de si la guerra es justa en cuanto a sus fines o no. Las naciones se consolidan y con ellas sus ejércitos. A partir del siglo xvi, la clase militar no sólo cumple un papel importante en la sociedad, sino que impone su propia concepción de la guerra como empresa con sus propias leyes y objetivos. Será von Clausewitz quien más adelante haga de esta concepción la primera teoría sistemática de la guerra.

Al igual que en el caso de la doctrina de la guerra justa, existen varias maneras de formular la llamada "doctrina militarista". Empero, podemos decir que, en general, todas esas versiones tendrían que incluir los tres principios que a continuacón intentaremos discutir:

1. Toda nación tiene el derecho a responder ataques enemigos.

2. Los soldados tienen la obligación de obedecer órdenes superiores.

3. Es legítima toda acción considerada como de estricta "necesidad" militar.

1. El primer principio tiene un carácter general y se refiere al derecho que tiene toda nación a defenderse de una agresión externa. No es de ningún modo un principio novedoso, Hobbes hizo clara referencia a este derecho "inherente" a la sobernía de las naciones (Leviathán, parte II, cap. 18, §9) y otros autores después de él han argumentado que si una nación es entendida como una estructura social y política cuya función es la de aglutinar y proteger a los individuos que la conforman, se sigue sin mayores dificultades el hecho de que ésta debe desarrollar los mecanismos suficientes para cumplir esa función. Resultaría paradójico que una nación no estuviera interesada en salvaguardar la unidad y el patrimonio que en buena medida la constituyen. El derecho a la guerra queda, sin embargo, condicionado a la circunstancia de que la nación, efectivamente, se vea en peligro. De donde se sigue que el aspecto más problemático del principio es la vaguedad del término "agresión". Una agresión será, sin duda, que un

5 Ibid., p. 38.

6 El concepto de "nación" y los relacionados con ella, tales como "nacionalismo" o "espiritu nacional" han sido objeto de estudios históricos y antropológicos muy relevantes. Entre los más importantes destacan los de Eric Hobsbawn, Naciones y nacionalismos y Benedict Anderson, Comunidades imaginadas. Aunque muy diferentes entre sí, ambos autores coinciden en afirmar que la idea de "nación" surge en la modernidad. El punto que me interesa señalar es que junto con la idea de "nación" también se consolida una nueva concepción acerca de la guerra cuyo eje central es la doctrina militarista. 
ejército avance más allá de las fronteras de un país con el objeto de ocupar el territorio; lo es también que aviones bombarderos ocupen espacio aéreo del enemigo y, por supuesto, que lancen sus bombas sin previo aviso. Pero no son estos los únicos casos en los que puede hablarse de "agresión", i.e., ésta no necesita ser tan clara para que una nación pueda invocar el derecho a responder dicha agresión. El principio podrá ser invocado también en el caso de guerras de agresión que no responden a algún tipo de ataque "bélico", esto es, dada la ambigüedad del término y de la imposibilidad de contar con una lista exhaustiva de "casos de agresión", si una nación está interesada en llevar a cabo una guerra que conviene a sus intereses económicos y políticos, podrá encontrar alguna manera de justificarse invocando este primer principio. (Recuérdese, por ejemplo, el caso de la guerra del Golfo Pérsico y los subsecuentes "correctivos" aplicados al pueblo iraquí).

Un problema práctico al que se enfrenta el primer principio de la doctrina militarista es que, a diferencia de lo que ocurrió en el pasado, el derecho de las naciones a declarar la guerra no sólo ha sido cuestionado en el presente siglo sino que se considera una violación a la sana convivencia entre las naciones, es decir, un crimen contra la paz. En efecto, a partir del Pacto de París (Kellogg-Briand) de 1928, el recurso de la guerra quedó proscrito como un recurso legítimo, como una solución a las controversias internacionales. El contenido del pacto marca oficialmente para las naciones firmantes el inicio de un nuevo orden, un jus contra bellum, que aunque no haya tenido resonancia efectiva suficiente como para impedir las agresiones militares entre las naciones, ha sentado un precedente de "no impunidad total" a este respecto. ${ }^{7}$

2. El segundo principio representa un punto ineludible en los manuales y códigos militares. Para que un ejército se constituya como tal deben establecerse jerarquías basadas en una obediencia estricta de los rangos inferiores hacia los superiores. La norma de conducta más importante que debe aprender un soldado es la obediencia absoluta hacia el superior inmediato y hacia las reglas que operan en ese complejo mundo que es un ejército. En el primer manual militar producido por Estados Unidos de Norteamérica (surgido durante la guerra civil de 1863 con ei título de General Orders 100) este principio fue introducido con el

7 El caso más reciente lo encontramos con el presidente serbio Milosevic. Si se logra juzgarlo, el primer cargo que tendrá que enfrentar es el que se refiere a crímenes contra la paz, y este servirá de base para acusarlo formalmente por crímenes de guerra y crímenes contra la humanidad. 
propósito de que los mandos intermedios y superiores del ejército pudieran tener control sobre la conducta de sus subordinados evitando así actos de pillaje y otro tipo de abusos cometidos contra la población civil. Sin embargo, está claro, que bajo esta fachada "moralista" del principio, la razón fundamental de su consideración es la posibilidad de garantizar que las órdenes militares, moralmente correctas o no, sean cumplidas al pie de la letra. En efecto, la parte débil del principio es que no garantiza que todas las órdenes dictadas por los mandos superiores sean órdenes justas o de acuerdo con los principios de proporción y discriminación. (Recordemos que son éstos, justamente, los principios que corresponden a la parte de la doctrina de la guerra justa conocida como jus in bello, el primero alude a que debe ser evitada la violencia y el sufrimiento excesivos, y el segundo se refiere a que debe respetarse a la población civil o, más ampliamente, a todo aquel que pueda ser considerado un "no-combatiente".) Por ejemplo, dada una situación en donde la orden constituye una violación a alguno de estos principios, el problema que se plantea es poder determinar qué tan responsable es el soldado por haber llevado a cabo dicha acción. Dicho de otra manera, la regla no toma en cuenta el hecho de que un mando superior cualquiera puede no tener claro, o incluso ignorar, qué tipo de acciones está prohibido. El problema con este segundo principio es, pues, que si bien está claro en cuanto a la obigación del soldado de obedecer órdenes superiores, no dice nada respecto de la responsabilidad de los agentes involucrados cuando una acción viola alguno de los principios que corresponden al jus in bello. ${ }^{8}$

3. Más complejo que los anteriores, el tercer principio intenta establecer la legitimidad de toda acción llevada a cabo según una estricta "necesidad militar". En realidad, este tercer principio constituye "la esencia" misma de la doctrina militarista: si la guerra es un asunto en el cual están en riesgo los intereses de una nación, toda acción dirigida a garantizar un resultado exitoso, será legítima. En la consecución de los fines de la guerra habrá, sin embargo, una cantidad muy variada de acciones y recursos que pueden ser empleados. Muchos recursos que consideraríamos violatorios de los principios de proporción y discriminación (jus in bello), y que constituyen, de acuerdo con las Convenciones de Ginebra, crímenes de guerra (por ejemplo el

8 La asimetría y ambigüedad entre los conceptos de "obediencia" y "responsabilidad" explica, en gran medida, el fracaso en el juicio contra los veinte oficiales y dos soldados citados por la comisión Peers, encargada de revisar los sucesos de Mi Lai y Son Mi durante la guerra de Vietnam. 
empleo de minas unipersonales), y crímenes contra la humanidad (persecuciones étnicas y genocidio) pueden ser defendidos en virtud de la mencionada "necesidad" militar. El problema que surge en relación con este principio es, sin duda, cómo definir el concepto de "necesidad militar". Según una larga tradición filosófica, "necesario" es un término que se aplica a un tipo especial de enunciados cuya característica definitoria es que no pueden ser falsos en ninguna circunstancia. Sobre este sentido del término no hay mayor controversia, empero, sí resulta problemático que a las acciones o a los hechos humanos se les confiera ese predicado: ¿Qué puede querer decir que una acción es necesaria en virtud de tal o cual objetivo? Lo primero que viene a la mente es la idea siguiente: una acción es necesaria si no dándose esa acción no se hubiera conseguido el objetivo en cuestión. Ahora bien, lo que habría que notar es que para que podamos llamar necesaria a una acción, el objetivo tuvo que haberse cumplido, i.e., es en relación con un objetivo alcanzado exitosamente que las acciones previas pueden considerarse necesarias. Es decir, es la parte vencedora a quien corresponde juzgar y valorar esas acciones: para el presidente Truman las bombas lanzadas sobre las islas japonesas constituyeron acciones necesarias para conseguir la rendición de Japón en la Segunda Guerra Mundial. Lo que en un principio pudo haberse considerado como desproporcionado respecto al objetivo que deseaba alcanzarse, quedó "plenamente justificado" al convertirse Estados Unidos en la potencia victoriosa. Pero ¿qué hubiera pasado si a pesar de las bombas lanzadas a Japón, las naciones del Eje hubieran resultado vencedoras en esa contienda? No hubiera podido disfrazarse la tremenda desproporción ( $y$, por ende, la inmoralidad) de dicha acción. De modo que el término "necesario", empleado en la jerga militarista, es un concepto tan cambiante y flexible como sólo pueden serlo los conceptos y criterios que dependen únicamente de la manera en que la persona que los fija, valora y concibe la situación.

\section{El problema en la aplicación de las leyes de la guerra. Conclusiones}

No es difícil reconocer que la doctrina militarista plantea serias dificultades a cualquier intento por introducir normas morales y, por ende, límites a las empresas bélicas. En la práctica ha quedado suficientemente constatado que es esta doctrina la que tiene preeminencia sobre las consideraciones morales en gran medida debido al hecho de que si se prestara atención a estas últimas no podrían cumplirse la mayoría de los objetivos y metas fijados por los intereses militares. La doctrina militarista descansa, así, en el supuesto de corte realista de que en la guerra no pueden hacerse concesiones si lo 
que se quiere es obtener la victoria. Si bien es cierto que en el juicio de Nuremberg se juzgó y dictó sentencia a aproximadamente 25 militares de distintos rangos por haber cometido crímenes de guerra y crímenes contra la humanidad, sería ingenuo pensar que más allá del "éxito" por las sentencias obtenidas logró consolidarse una normatividad de guerra para casos futuros. Ninguna conferencia, congreso o tribunal de guerra ha contado con la fuerza suficiente como para establecer claramente en quiénes y en qué circunstancias se vulneran los principios de humanidad que, supuestamente, deben prevalecer en las empresas bélicas. En todas las guerras de nuestro siglo (por hablar solamente de este periodo de la historia) se han violado sistemáticamente los principios teóricos de discriminación y proporción y es muy probable que esto siga sucediendo.

Así, una primera conclusión a la que podemos llegar es que el verdadero problema de las leyes de guerra no es si éstas existen "formalmente" sino si existen mecanismos (más allá de los ya probados) que pudieran garantizar su aplicación. En efecto, el problema de la aplicación de las leyes de guerra es que no existen los instrumentos o mecanismos independientes que puedan tener la fuerza suficiente para imponer restricciones a las acciones militares, tanto de perdedores como de victoriosos. A pesar de la ambigüedad de muchas de las reglas y convenciones contenidas en el derecho internacional, éste cuenta teóricamente con los elementos suficientes para identificar los abusos y crímenes que cometen las naciones en guerra. La cuestión es que dichas leyes se convierten en letra muerta cuando se trata de aplicarlas a las naciones más poderosas militarmente hablando.

Sabemos qué puede hacerse y qué no debe hacerse. Los no combatientes deberían ser respetados, ciudades indefensas no deberían ser bombardeadas, el sufrimiento innecesario debería ser evitado [...] Pero mientras las náciones soberanas sigan considerando como derecho inalienable la defensa de sus fines y ellas mismas sean los únicos jueces de dichos fines, ningún manual que pueda escribirse podrá servir de guía para los soldados que combaten... ${ }^{9}$

En efecto, no es demasiado difícil reconocer los casos en los que se cometen crímenes de guerra en todas sus variantes. La historia del presente siglo está plagada de ejemplos. Si se reconocen es porque, en principio, podemos hablar de "leyes de guerra". El punto importante que hay que destacar es que parece que estamos condenados a admitirlos como hechos corrientes. La tolerancia y la impunidad respecto de dichas acciones ha ido creciendo conforme se ha ido incrementando el poder de los armamentos. Pero el círculo tiene que romperse en algún punto y ese punto podría ser, paradójicamente, la propia doctrina militarista. Si alguna ventaja puede tener el

9 Donald A. Wells, War Crimes and the Laws of War, p. 150. 
desarrollo tecnológico aplicado a la empresa de la guerra, este desmedido poder de destrucción garantizado por el arsenal en manos de unas cuantas naciones, es que actúa no a favor, sino en contra de su antigua aliada la doctrina militarista. El defensor de esta doctrina se ve obligado a tomar posturas más moderadas en cuanto a los medios utilizados en las guerras. Ninguna guerra puede llevarse "hasta sus últimas consecuencias" por razones obvias. Y si es imposible mantener posturas militaristas extremas frente al arsenal bélico con que se cuenta en la actualidad, el siguiente paso es pugnar porque la guerra se conciba como un actividad llevada a cabo por sujetos morales y no a la inversa, i.e., no como se ha pretendido desde la doctrina militarista: ajustar la moral a los propósitos y fines de la guerra.

Cualquiera que sea el objetivo al que se aspira y los medios empleados para conseguirlo, la demanda de necesidad militar tiene que ser enfocada junto con otros dos requisitos que surgen de la propia naturaleza del hombre en tanto ser moral y social. El primero impone un principio ético: uno no deja de ser un ser moral al tomar las armas aun cuando por un requisito de necesidad moral se cometan actos inmorales [...] El segundo impone un principio prudencial: uno no debe comportarse con el enemigo de modo tal que una futura reconciliación sea imposible. ${ }^{10}$

Como tampoco debería de resultar imposible, aunque habría que pensar más en ello, que esta postura pudiera ser incompatible con una tarea más a largo plazo que sería, evidentemente, la del destierro definitivo de la guerra del panorama futuro de la humanidad.

BIBLIOGRAFIA

Elshtain, Jean B. (ed.), Just War Theory, New York University Press, Nueva York, 1992.

Gallois, Roger, La cuesta de la guerra, Fondo de Cultura Económica, México, 1975. Norman, Richard, Ethics Killing and War, Cambridge University Press, Nueva York, 1995.

Walzer, Michael, Just and Unjust Wars, Basic Books, Library of Congress, Estados Unidos, 1977.

Wells, Donald A., War Crimes and the Laws of War, University Press of America, Estados Unidos, 1991.

Recibido: 23 de junio de 1999

10 Michael Howard, “Can War Be Controlled?”, en J.B. Elshtain (ed.), op. cit. . 